\title{
CONCERNING SPECIAL CENTERS OF PROJECTION FOR AN ALGEBRAIC SPACE BRANCH*
}

\author{
BY W. S. TURPIN
}

1. Introduction. If one projects a branch of an algebraic space curve from a point $(a, b, c)$ onto a plane and considers $a, b$, and $c$ as parameters, one obtains a plane branch possessing a development whose coefficients are rational functions of $a, b$, and $c$. If it now be assumed that no relations exist between the parameters $a, b$, and $c$, this plane branch will have a certain generic composition which will change only for special values of these parameters which satisfy certain relations between the coefficients of the development. A center of projection is said to be generic with respect to a space branch and the corresponding plane projection to be a generic projection, provided the latter has a generic composition in the sense just defined.

Until recently, it had been thought that the composition of a space branch was the same as that of its generic plane projection. $\dagger$ However, it has been shown by example that the composition of a space branch is not necessarily the same as that of its generic projection. $f$

In view of this it was deemed of interest to investigate the conditions under which a center of projection is generic with respect to a given space branch. In what follows, these conditions are determined and an explicit formulation for the locus of nongeneric centers of projection is given.

2. A Theorem on Plane Branches. It will be convenient first to establish a theorem concerning the composition of a plane branch. The equation of such a branch, with origin at the origin of coordinates, may be written in the following manner :§

* Presented to the Society, April 11, 1936.

$\uparrow$ See, for instance, Enriques-Chisini, Lezioni sulla Teoria Geometrica delle Equazioni e delle Funzioni Algebriche, vol. 2, p. 559.

$\ddagger$ O. Zariski, Algebraic Surfaces, Ergebnisse der Mathematik und ihrer Grenzgebiete, vol. 3, no. 5, pp. 11-12.

$\S$ For a detailed discussion concerning such parametric representations, see Enriques-Chisini, op. cit., vol. 2, p. 330 et seq. The notation used here is due to Zariski: O. Zariski, op. cit., p. 7. 


$$
\begin{aligned}
x= & \tau^{\nu} \\
y= & \sum_{1}^{k_{1}} a_{1 i} \tau^{\nu_{\imath}}+b_{1} \tau^{m_{1} \nu_{2}}+\cdots+\sum_{1}^{k_{g}} a_{g i} \tau^{\left(m_{g-1}+i\right) \nu_{\imath}}+b_{g} \tau^{m_{g}} \\
& +\sum_{1}^{\infty} c_{i} \tau^{m_{g}+i} \\
= & {\left[\tau: a, b ; m_{1}, n_{1}, \cdots, m_{g}, n_{g}\right], }
\end{aligned}
$$

where

$$
\begin{aligned}
& \nu=n_{1} n_{2} \cdots n_{g} ; \nu_{g+1}=1 ; m_{0}=1 ; \nu_{j}=n_{j} n_{j+1} \cdots n_{g} ; \quad b_{j} \neq 0 ; \\
& \left(m_{j}, n_{j}\right)=1 ; \quad k_{j}=\left[\frac{m_{j}-m_{j-1} n_{j}}{n_{j}}\right], \quad(j=1,2, \cdots, g) .
\end{aligned}
$$

Then $b_{j} \tau^{m^{i} u^{j+1}}(j=1,2, \cdots, g)$ are said to be the characteristic terms of the development of the branch, the exponents $m_{j} \nu_{j+1}$ are said to be the characteristic exponents of the branch, and the pairs $\left(m_{j}, n_{j}\right)$ are said to be the characteristic pairs of the branch.

Let us now consider a branch of the form :

$$
\begin{aligned}
X= & \sum_{i=1}^{k_{1}} a_{1 i}^{(1)} t^{\nu_{i}}+b_{1}^{(1)} t^{m_{1} \nu_{2}}+\cdots+\sum_{i=1}^{k_{g}} a_{g i}^{(1)} t^{\left(m_{g-1}+i\right) \nu_{g}} \\
& +b_{g}^{(1)} t^{m_{g}}+\sum_{i=1}^{\infty} c_{i}^{(1)} t^{m_{g}+i} \\
Y= & \sum_{i=1}^{k_{1}} a_{1 i}^{(2)} t^{\nu_{i}}+b_{1}^{(2)} t^{m_{1} \nu_{2}}+\cdots+\sum_{i=1}^{k_{g}} a_{g i}^{(2)} t^{\left(m_{g-1}+i\right) \nu_{g}} \\
& +b_{g}^{(2)} t^{m_{\theta}}+\sum_{i=1}^{\infty} c_{i}^{(2)} t^{m_{g}+i}
\end{aligned}
$$

where $a_{11}{ }^{(j)} \neq 0 \neq b_{i}{ }^{(j)}$ for $j=1,2$ and $i=1,2, \cdots, g$.

We prove the following theorem:

Theorem. The pairs $\left(m_{j}, n_{j}\right)$ are characteristic pairs of the branch (2) if and only if

$$
\left|\begin{array}{cc}
a_{11}^{(1)} & b_{j}^{(1)} \\
a_{11}^{(2)} & b_{j}^{(2)}
\end{array}\right| \neq 0, \quad(j=1,2, \cdots, g) .
$$

Proof: Let us make a change in parameter, setting $X=\tau^{\nu}$. 
Then we have $t=\alpha \tau+\cdots$, where $\alpha \neq 0$. We first prove that $t \tau^{\nu-1}=\left[\tau ; \alpha, \beta ; m_{1} n_{1} \cdots m_{o} n_{0}\right]$ where $\alpha_{11}=\alpha \neq 0 \neq \beta_{j}$.

Let $t \tau^{\nu-1}=\phi(\tau)$. We first notice that the leading term in $\tau^{\nu}$ must occur in $\phi(\tau)$. Let us now suppose $\delta_{j} \tau^{l_{j}}$ is the first term of the power series $\phi(\tau)$ whose exponent is not divisible by $\nu_{j}$. Then we have

$$
\begin{aligned}
t^{\nu}= & \left(\text { terms whose exponents are divisible by } \nu_{j}\right) \\
& +(\nu-1) \alpha_{11}^{\nu-1} \delta_{j} \tau^{l_{j}} \\
& +(\text { terms of higher degree in } \tau)
\end{aligned}
$$

and, in fact, for any positive integer $\sigma$,

$$
\begin{aligned}
t^{\nu_{j} \sigma}= & \left(\text { terms whose exponents are divisible by } \nu_{j}\right) \\
& +\left(\nu_{j} \sigma-1\right) \alpha_{11}^{\nu_{j} \sigma-1} \delta_{j} \tau^{\nu\left(\nu_{j} \sigma-\nu\right)+l_{j}} \\
& + \text { (terms of higher degree). }
\end{aligned}
$$

We also have

$$
b_{j}^{(1)} t^{m_{j} v_{j+1}}=b_{j}^{(1)} \alpha_{11}^{m_{j} v_{j+1}} \tau^{m_{j} \nu_{j+1}}+\text { (terms of higher degree). }
$$

The contribution of a term $t^{\nu_{j} \sigma}$ gives for the first term whose exponent is not divisible by $\nu_{j}$ one whose exponent is $\geqq l_{j}$ and the equality sign holds only for $\nu_{j} \sigma=\nu$. Since $X=\tau^{\nu}$, the contribution $(\nu-1) \alpha_{11}^{\nu-1} \delta_{j} \tau^{l_{j}}$ must cancel out. It cannot cancel out with any term arising from the contribution of $t^{\nu j}$ since it is the term of lowest degree whose exponent is not divisible by $\nu_{j}$. Therefore, $l_{j} \geqq m_{j} \nu_{j+1}$. Also, the contribution $b_{j}^{(1)} \alpha_{11}^{m_{j} \nu_{j+1}} \tau^{m_{j} \nu_{j+1}}$ must cancel out. Now it cannot cancel with any term whose exponent is less than $l_{j}$ since all such terms have exponents which are divisible by $\nu_{j}$. Consequently, $m_{j} \nu_{j+1} \geqq l_{j}$. On combining these two inequalities, we have the relation $l_{j}=m_{j} \nu_{j+1}$, and the statement is established.

On substituting this result in the development for $Y$, it is seen that the first term which occurs whose exponent is not divisible by $\nu_{j}$ is a term in $\tau^{m_{j} \nu_{j+1}}$ provided that its coefficient

$$
B_{j}^{(2)}=(\nu-1) a_{11}^{(2)} \alpha_{11}^{\nu-1} \beta_{j}+b_{j}^{(2)} \alpha_{11}^{m_{j} \nu_{j+1}}
$$

is distinct from zero.

Thus $\left(m_{j}, n_{j}\right)$ is a characteristic pair of the branch (2) if and only if $B_{j}^{(2)} \neq 0$. 
Now the development for $X$ must reduce to $\tau^{\nu}$ and we must thus have the relation

$$
B_{j}^{(1)}=(\nu-1) a_{11}^{(1)} \alpha_{11}^{\nu-1} \beta_{j}+b_{j}^{(1)} \alpha_{11}^{m_{j} \nu_{j+1}}=0 .
$$

It therefore follows that $\left(m_{j}, n_{j}\right)$ is a characteristic pair of (2) if and only if the system of equations

$$
\left\{\begin{array}{l}
B_{j}^{(1)}=0 \\
B_{j}^{(2)}=0
\end{array}\right\}
$$

possesses no solution. The system (B) cannot be satisfied by the trivial solution since $\alpha_{11} \neq 0 \neq \beta_{j}$. Consequently, (B) possesses no solution if and only if

$$
\Delta_{j}=\left|\begin{array}{cc}
a_{11}^{(1)} & b_{j}^{(1)} \\
a_{11}^{(2)} & b_{j}^{(2)}
\end{array}\right| \neq 0 .
$$

This establishes the theorem.

3. Projections of Space Branches. Let us now consider a space branch

$$
\begin{aligned}
& x=t^{\nu}, \\
& y=f(t)=a t^{\nu}+\cdots, \\
& z=\phi(t)=b t^{\nu}+\cdots .
\end{aligned}
$$

If we project such a branch from a point $\left(x_{0}, y_{0}, z_{0}\right)$ onto a plane, say $z=0$, and consider $x_{0}, y_{0}$, and $z_{0}$ as parameters, we obtain a plane branch

$$
\begin{aligned}
& x=x\left(t ; x_{0}, y_{0}, z_{0}\right), \\
& y=y\left(t ; x_{0}, y_{0}, z_{0}\right),
\end{aligned}
$$

in which the coefficients are rational functions of $x_{0}, y_{0}, z_{0}$ (in fact, polynomials in $x_{0}$ and $y_{0}$ ). If we assume that no relations exist between the parameters, (4) will have order $\nu$ and certain characteristic pairs $\left(m_{j}, n_{j}\right)$. These pairs will cease to be characteristic only for special positions of the point $\left(x_{0}, y_{0}, z_{0}\right)$ for which the coefficients of the characteristic terms vanish. When no such special relations hold, the center of projection is evidently generic. Obviously, the non-generic centers of projection for a given branch fill up certain algebraic spreads; we shall proceed to the explicit determination of these spreads. 
Let us assume that the points at infinity on the three axes are generic with respect to the branch(3). Then the three parallel projections of this branch are generic and we may write its development in the form:

$$
\begin{aligned}
x= & t^{\nu}, \\
y= & \sum_{i=1}^{k_{1}} a_{1 i} t^{\nu_{i}}+b_{1} t^{m_{1} \nu_{2}}+\cdots+\sum_{i=1}^{k_{g}} a_{g i} t^{\left(m_{g-1+i} \nu_{g}\right.}+b_{g} t^{m_{g}} \\
& +\sum_{i=1}^{\infty} c_{i} t^{m_{g+i}}, \\
z= & \sum_{i=1}^{k_{1}} \alpha_{1 i} t^{\nu_{i}}+\beta_{1} t^{m_{1} \nu_{2}}+\cdots+\sum_{i=1}^{k_{g}} \alpha_{g i} t^{\left(m_{g-1+i}+\nu_{g}\right.}+\beta_{g} t^{m_{g}} \\
& +\sum_{i=1}^{\infty} \gamma_{i} t^{m_{\theta}+i}
\end{aligned}
$$

where, by the preceding section, $\alpha_{11} \neq 0 \neq a_{11}, \beta_{j} \neq 0 \neq b_{j}$,

$$
\left|\begin{array}{cc}
a_{11} & b_{j} \\
\alpha_{11} & \beta_{i}
\end{array}\right| \neq 0, \quad(j=1,2, \cdots, g) .
$$

Let us project the branch (5) from the point $\left(x_{0}, y_{0}, z_{0}\right)$ onto the plane $x=0$. Assume $x_{0} \neq 0$, for in the case $x_{0}=0$ the projection is degenerate. The projecting cone is given by

$$
\begin{gathered}
X=\frac{\rho x_{0}+\sigma x}{\rho+\sigma}, \\
Y=\frac{\rho y_{0}+\sigma y}{\rho+\sigma}, \\
Z=\frac{\rho z_{0}+\sigma z}{\rho+\sigma} .
\end{gathered}
$$

Cutting this cone by the plane $X=0$, we obtain the relations

$$
\rho x_{0}+\sigma x=0 ; \quad \rho=-\frac{\sigma x}{x_{0}}=-\frac{\sigma t^{\nu}}{x_{0}} .
$$

Therefore, the projection of (5) from the point $\left(x_{0}, y_{0}, z_{0}\right)$ onto the plane $x=0$ is given by the equations 
(8)

$$
\begin{aligned}
y & =\frac{\left[t ; a, b ; m_{1} n_{1} \cdots m_{g} n_{g}\right]}{1-\frac{t^{\nu}}{x_{0}}} \\
& =\left[t ; a, b ; m_{1} n_{1} \cdots m_{g} n_{g}\right] \cdot\left(1+\frac{t^{\nu}}{x_{0}}+\frac{t^{2 \nu}}{x_{0}^{2}}+\cdots\right), \\
z & =\frac{\left[t ; \alpha, \beta ; m_{1} n_{1} \cdots m_{g} n_{g}\right]}{1-\frac{t^{\nu}}{x_{0}}} \\
& =\left[t ; \alpha, \beta ; m_{1} n_{1} \cdots m_{g} n_{g}\right] \cdot\left(1+\frac{t^{\nu}}{x_{0}}+\frac{t^{2 \nu}}{x_{0}^{2}}+\cdots\right) .
\end{aligned}
$$

We now apply our theorem on plane branches. The characteristic pairs of the projection (8) are accordingly $\left(m_{j}, n_{j}\right)$ if and only if

$$
D_{j}=\left|\begin{array}{cc}
a_{11}-\frac{y_{0}}{x_{0}} & b_{j} \\
\alpha_{11}-\frac{z_{0}}{x_{0}} & \beta_{j}
\end{array}\right| \neq 0, \quad(j=1,2, \cdots, g) \text {. }
$$

Thus we have shown that a necessary and sufficient condition that the projection (8) of (5) be generic is that $D_{j} \neq 0$ for $j=1,2, \cdots, g$. It therefore follows that the point $\left(x_{0}, y_{0}, z_{0}\right)$ is a generic center of projection for the space branch (5) provided it is not incident to any one of the $g$ planes

$$
P_{j}:\left|\begin{array}{lll}
x & y & z \\
1 & a_{11} & \alpha_{11} \\
0 & b_{j} & \beta_{j}
\end{array}\right|=0, \quad(j=1,2, \cdots, g) .
$$

The planes $P_{j}$ all pass through the tangent line of the branch (5). The osculating plane of (5) is a member of this set if and only if the determinants

$$
\left|\begin{array}{cc}
a_{11} & \alpha_{11} \\
a_{1 j} & \alpha_{1 j}
\end{array}\right|, \quad\left(j=2,3, \cdots, k_{1}\right)
$$

all vanish.

The Johns Hopkins University 